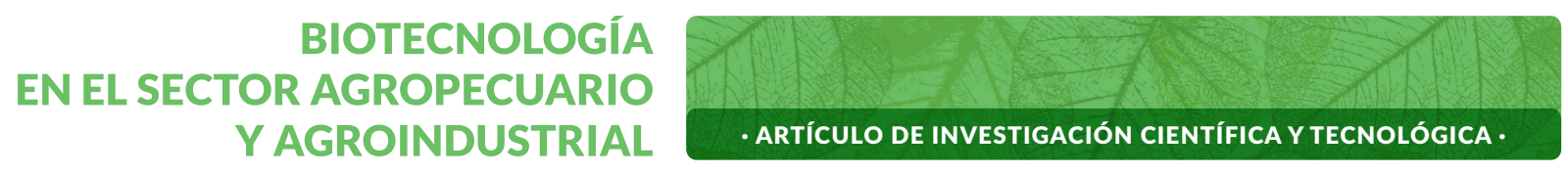

\title{
Clonación del cDNA codificante de las subunidades de la gonadotropina luteinizante de Pimelodus grosskopfii
}

\section{Cloning of the cDNA coding for the luteinizing gonadotropin subunits of Pimelodus grosskopfii}

\section{Clonagem molecular do cDNA codificante de las subunidades da gonadotropina luteinizante de Pimelodus grosskopfii}

JAMES BETANCUR-LÓPEZ1 MARÍA FERNANDA FLÓREZ², ANA MARÍA PÉREZ-O’BRIEN, NEIL VASQUEZ-ARAQUE ${ }^{4}$, ANDRÉS MONTOYA-LÓPEZ

\footnotetext{
Historial del Artículo

Recibido para evaluación: 24 de Febrero 2020.

Aprobado para publicación: 13 de Mayo 2020.

1 Universidad Nacional de Colombia, Facultad de Ciencias, Grupo de Biotecnología Animal. Zootecnista. Medellín, Colombia.

2 Asociación Colombiana de Acuicultores (ASOACUICOLA). MSc Microbióloga. Medellín, Colombia. ORCID: https://orcid.org/0000-0001-6945-5212

3 Asociación Colombiana de Acuicultores (ASOACUICOLA). Médica veterinaria zootecnista, PhD. Medellín, Colombia

4 Universidad Nacional de Colombia, Facultad de Ciencias, Grupo de Biotecnología Animal. Doctor en Biotecnología. Medellín, Colombia

5 Asociación Colombiana de Acuicultores (ASOACUICOLA). Zootecnista, Doctor en Ciencias Animales. Medellín, Colombia.
}

Correspondencia: jjbetancurl@unal.edu.co 


\section{RESUMEN}

Pimelodus grosskopfii, es un Siluriforme importante en la pesca continental en Colombia y de interés en la acuicultura. La presente investigación tuvo como objetivo clonar y determinar las secuencias codificantes de las subunidades GP- $\alpha$ y LH $\beta$ de la gonadotropina luteinizante de esta especie. Para lograr lo anterior, el ARN total de glándulas pituitarias fue extraído y se realizó síntesis de cDNA. Los productos de PCR purificados de GP- $\alpha$ y LH $\beta$ fueron ligados en $p J E T 1.2 /$ blunt ${ }^{\circledR}$ seguido de la transformación de Escherichia coli $D H 5 \alpha^{\circledR}$ y posterior secuenciación de fragmentos de interés. El marco abierto de lectura de las subunidades GP- $\alpha$ y LH $\beta$ fue de 351 pb (116 aminoácidos) y $423 \mathrm{pb}$ (140 aminoácidos), respectivamente. Se encontró una alta homología (aminoácidos) en la subunidad GP- $\alpha$ con otras familias de Siluriformes (8799\%) y menor homología con órdenes como Perciformes, Salmoniformes y Cypriniformes (66-90\%). De igual manera para la subunidad LH 3 , la homología (aminoácidos) fue alta al interior del orden Siluriformes (91-97\%) y menor con otros ordenes (65-86\%). Los resultados obtenidos en el presente estudio ofrecen información básica sobre las secuencias de las gonadotropinas en bagres neotropicales y pueden ser utilizados para el desarrollo de hormonas recombinantes para optimizar el control reproductivo en P. grosskopfii.

\section{ABSTRACT}

Pimelodus grosskopfii is an important catfish in inland fisheries in Colombia as well as an attractive species for aquaculture. The aim of this study was to clone and determine the coding sequence of the GP- $\alpha$ and LH $\beta$ subunits of the luteinizing gonadotropin of this species. Total RNA from pituitary glands was extracted and CDNA was synthesized. GP- $\alpha$ and LH $\beta$ purified $P C R$ products were linked to pJET1.2/blunt ${ }^{\circledR}$ followed by Escherichia coli $\mathrm{DH} 5 \alpha \mathbb{R}$ transformation. The extracted plasmids of the positive clones were sequenced. The open reading frame of GP- $\alpha$ and LH $\beta$ subunits was $351 \mathrm{bp}$ (116 amino acids) and 423 bp (140 amino acids) respectively. The GP- $\alpha$ subunit showed high degrees of homology (amino acids) with GP- $\alpha$ subunit from different families of Siluriformes (87-99\%) and lower homology with teleost orders such as Perciformes, Salmoniformes and Cypriniformes (66$90 \%)$. Similarly, the homology (amino acids) of LH $\beta$ subunit was high within siluriformes (91-97\%) and lower with other orders (65-86\%). The results obtained in the present study, provide basic information on the sequence of gonadotropins in neotropical catfishes and can be used for the development of recombinant hormones to optimize reproductive control in P. grosskopfii.

\section{PALABRAS CLAVE:}

Glicoproteínas, Secuencia codificante, Siluriformes.

\section{KEYWORDS:}

Coding sequence, Glycoproteins, Siluriformes

\section{PALAVRAS-CHAVE:}

Glicoproteínas, Sequência de codificação, Siluriformes.

Cómo citar este artículo: JAMES BETANCUR-LÓPEZ, MARÍA FERNANDA FLÓREZ, ANA MARÍA PÉREZ-O’BRIEN, NEIL VASQUEZ-ARAQUE, ANDRÉS MONTOYALÓPEZ. Clonación del cDNA codificante de las subunidades de la gonadotropina luteinizante de Pimelodus grosskopfii. Revista Biotecnología en el Sector Agropecuario y Agroindustrial,18(2),20.37-47, DOI:http://dx.doi.org/10.18684/BSAA(18)37-47 


\section{RESUMO}

Pimelodus grosskopfii é um peixe importante nas pescarias de agua doce da Colômbia, além de ser uma espécie potencial para a aquicultura. $O$ objetivo deste estudo foi clonar e determinar a sequência de codificação das subunidades GP- $\alpha$ e LH $\beta$ da gonadotrofina luteinizante de P grosskopfii. O RNA total das glândulas pituitárias foi extraído e 0 cDNA foi sintetizado. Os produtos de PCR purificados de GP- $\alpha$ e LHp foram ligados a pJET1.2 / blunt $\AA$, seguido pela transformação de Escherichia coli $\mathrm{DH} 5 \alpha{ }^{\circledR}$ e sequenciamento dos plasmídeos extraídos dos clones positivos. A grelha de leitura aberta das subunidades GP- $\alpha$ e LH $\beta$ foi de 351 pb (116 aminoácidos) e 423 pb (140 aminoácidos), respectivamente. A subunidade GP- $\alpha$ apresentou altos graus de homologia (aminoácidos) com a subunidade GP- $\alpha$ de diferentes famílias de Siluriformes (87-99\%) e menor homologia com ordens de teleósteos, como Perciformes, Salmoniformes e Cypriniformes (66-90\%). Da mesma forma, a homologia (aminoácidos) da subunidade LH $\beta$ foi alta com os Siluriformes (91-97\%) e menor com outras ordens (65-86\%). Os resultados obtidos no presente estudo fornecem informações básicas sobre a sequência das gonadotrofinas em bagres neotropicais e podem ser utilizados para o desenvolvimento de hormônios recombinantes para otimizar o controle reprodutivo em P. grosskopfii.

\section{INTRODUCCIÓN}

Las hormonas glicoproteínas (GPHs) son una familia de moléculas ricas en cisteínas que se han conservado evolutivamente. Entre las GPHs, se encuentran las hormonas gonadotropinas (GTH) como la hormona folículo estimulante $(\mathrm{FSH})$ y la hormona luteinizante (LH), también denominadas en la literatura de peces teleósteos como GTH I y GTH II respectivamente. Las GTHs, contienen dos subunidades disimiles designadas como $\alpha$ y $\beta$ codificadas por genes diferentes [1]; mientras la subunidad $\alpha(G P-\alpha)$ es la misma entre gonadotropinas de una especie, la subunidad $\beta$ (FSH $\beta$ o LH $\beta$ ) es única para cada hormona y le confiere especificidad biológica [2]. Las estructuras de los de genes de GP- $a$ y FSH $\beta$ se han conservado durante la evolución entre peces teleósteos y mamíferos. En contraste, los loci del gen $\mathrm{LH} \beta$ de los teleósteos y los tetrápodos no muestran ninguna relación sintética [3].

Las funciones de la LH en la activación de la maduración de los ovocitos y la ovulación parecen ser similares entre los vertebrados [4]. En el modelo de teleósteos mejor estudiado que es el pez cebra, la LH mediante la señalización de AMPc regula la expresión de la proteína reguladora aguda esteroidogénica (star), la cual interviene en la maduración de los ovocitos catalizando el primer paso en la síntesis de la hormona inductora de la maduración (MIH) [5]. Por otro lado, en los machos de pez cebra la señalización de las gonadotropinas es esencial para la espermatogénesis y espermiación, sin embargo las acciones de la LH y FSH están altamente superpuestas [4].

Los bagres de la familia Pimelodidae son diversos y abundantes en la vasta red de ríos de tierras bajas que fluyen desde los Andes y los escudos continentales hacia el Océano Atlántico, en la cuenca del Maracaibo y en la vertiente del Pacífico de Panamá [6,7]. Diferentes especies de pimelodidos como Pseudoplatystoma spp., Leiarius spp, Brachyplatystoma spp y Pimelodus spp. son económicamente importantes en las pesquerías continentales de Suramérica y algunas poseen un alto potencial para la acuicultura. Muchos pimelodidos realizan migraciones reproductivas sincronizadas con el ciclo hidrológico [8,9]. Por esta razón, se presenta una disrupción reproductiva en cautiverio debido a la falta de estímulos ambientales apropiados y que en los machos de Pimelodus spp. se agrava porque los testículos digitiformes [10] impiden que el semen se libere por masaje abdominal durante los procedimientos de reproducción inducida [11]. Por otro lado, la ausencia tanto de maduración final de los ovocitos como de ovulación en pimelodidos en cautiverio, indica que el desove parece estar estrictamente modulado por la síntesis y liberación de LH $\beta$ [12].

Pimelodus grosskopfii es un pez críticamente amenazado, de importancia comercial en las pesquerías artesanales y endémico de las cuencas de los ríos Magdalena, Cauca y San Jorge [13,14]. Al igual que en las demás especies de la familila Pimelodidae, las secuencias codificantes de las GTH de P. grosskopfii son desconocidas. El objetivo de la presente investigación fue aislar, clonar y determinar las secuencias de las subunidades GP- $a$ y LH $\beta$ de la LH de P. grosskopfii para generar información básica de las gonadotropinas como requisito importante para estudios de regulación de la gametogénesis en Siluriformes tropicales y como insumo fundamental en la síntesis de hormonas recombinantes especie-específicas que mejoren la eficiencia reproductiva en cautiverio de esta especie. 


\section{MÉTODO}

\section{Material biológico}

Individuos adultos de Pimelodus grosskopfii (250-300 g) fueron obtenidos en el Centro Acuícola Piscícola Santa Cruz (Caucasia, Antioquia). Los peces fueron sacrificados mediante inmersión en una solución de MS-222 a una concentración de 300 ppm. Posteriormente, las glándulas pituitarias fueron extraídas y almacenadas en RNAlater ${ }^{\mathrm{TM}}$ (QIAGEN) a $-80^{\circ} \mathrm{C}$, hasta laextracción de ARN.

\section{Extracción de ARN y transcripción reversa}

El procesamiento de las muestras se realizó en el laboratorio de Biología Molecular de la Asociación Colombiana de Acuicultores en Medellín, Colombia.

Las pituitarias fueron congeladas en nitrógeno líquido y pulverizadas con un homogenizador en solución de lisis (QIAGEN). El ARN total fue extraído en el sistema automatizado QIAcube (QIAGEN) empleando la programación para extracción a partir de tejidos con el Kit RNeasy Mini (QIAGEN). La integridad del ARN fue verificada mediante electroforesis de geles de agarosa $1,5 \%$ en TBE $0,5 \mathrm{X}$ y basado en la absorbancia a 260 nm empleando el Nanodrop 2000 (Thermo Scientific).

La síntesis de la primera cadena de cADN se realizó empleando el Kit RevertAid H Minus First Strand cDNA Synthesis (ThermoScientific) usando Oligo (dT) y cebadores random hexamer. La transcripción reversa se llevó a cabo por $60 \mathrm{~min}$ a $42^{\circ} \mathrm{C}$ en una reacción de $20 \mu \mathrm{L}$, con $1 \mu \mathrm{g}$ de ARN, $4 \mu \mathrm{L} 5 \mathrm{X}$ de búfer de reacción, 0,5 mM mezcla dNTP, $20 \mathrm{U}$ RNase Inhibitor, $200 \mathrm{U}$ RevertAid $\mathrm{H}$ Minus y finalización a $70^{\circ} \mathrm{C}$ por $5 \mathrm{~min}$. El producto de la reacción fue almacenado a $-80^{\circ} \mathrm{C}$ hasta su uso en la PCR.

\section{Amplificación GP- $\alpha$ y LH $\beta$ mediante PCR}

Se diseñaron 13 diferentes grupos de cebadores a partir de las secuencias de las subunidades de la LH de otras especies de Siluriformes depositadas en las bases de datos de NCBI (National Center for Biotechnology Information) (cuadro 1). Para GP- $a$, se obtuvo amplificación con dos grupos de cebadores denominados como 3GP-a y 4GP-a. El perfil térmico utilizado para estas PCR fue desnaturalización inicial a $94^{\circ} \mathrm{C}$ por dos minutos, 35 ciclos de $94^{\circ} \mathrm{C}$ por un minuto, $49,1^{\circ} \mathrm{C}$ por 30 segundos, $72^{\circ} \mathrm{C}$ por 40 segundos y una extensión final a $70^{\circ} \mathrm{C}$ durante 5 minutos. Para la subunidad LH $\beta$, se obtuvo amplificación con los cebadores nombrados como $7 \mathrm{LH} \beta$. El perfil térmico utilizado fue desnaturalización inicial a $94^{\circ} \mathrm{C}$ por 2 minutos, 35 ciclos de $94^{\circ} \mathrm{C}$ por un minuto, $58^{\circ} \mathrm{C}$ por 30 segundos y $72^{\circ} \mathrm{C}$ por 40 segundos y una extensión final a $70^{\circ} \mathrm{C}$ durante 5 . Para todas las PCR, las concentraciones de reactivos fueron: $2 \mathrm{mM}$ $\mathrm{MgSO}_{4}, 0,2 \mathrm{mM}$ dNTP's, 0,5 $\mu \mathrm{M}$ cebadores, 2,5 U Pfu DNA Polymerase (Thermo Scientific).

\section{Clonación, transformación y purificación}

Los productos de la PCR de las subunidades GP- $a$ o $\mathrm{LH} \beta$ fueron clonados en el vector pJET1.2/blunt (Life Technologies). Las condiciones de la reacción de clonación fueron: $10 \mu \mathrm{L}$ de búfer de reacción (2X), $1 \mu \mathrm{L}$ pJET1.2/blunt (50 ng/ $\mu \mathrm{L}), 1 \mu \mathrm{L}$ T4 DNA ligasa $(5 \mathrm{U} / \mu \mathrm{L}$ ), $7 \mu \mathrm{L}$ Agua libre de nucleasas, $1 \mu \mathrm{L}$ producto de PCR. La reacción de clonación se incubó a $20^{\circ} \mathrm{C}$ por una hora. La transformación de Escherichia coli DH5a (Thermo Scientific), se realizó por choque térmico, empleando 50 $\mu \mathrm{L}$ de células competentes y $5 \mu \mathrm{L}$ del constructo (vector pJET 1.2/blunt con producto de PCR GP-a ó LH $\beta$ ), siguiendo las recomendaciones de la casa comercial.

La recuperación del constructo se realizó a partir de colonias transformadas, que se inocularon en medio LB con Ampicilina a $37^{\circ} \mathrm{C}$ en agitación a $250 \mathrm{rpm}$ por 16 horas. La purificación se realizó con el sistema Miniprep QIAprep Spin (QIAGEN). Posteriormente, se realizó la verificación de la clonación mediante una nueva PCR para cada subunidad.

\section{Secuenciación}

Una vez realizada la purificación de los constructos con la inserción de cada una de las subunidades, los clones identificados como positivos fueron enviados a Macrogen (Corea), para realizar secuenciamiento en ambas direcciones. Las secuencias obtenidas fueron editadas manualmente en el programa BioEdit versión 7.2.5. La creación de las secuencias consenso fue realizado en el programa Geneious versión 5.4.6. La comparación de las secuencias de GP-a y $\mathrm{LH} \beta$ con las depositadas en GenBank se realizó mediante BLAST con las herramientas en línea de NCBI. 
Cuadro 1. Cebadores diseñados para amplificar secuencias de GP-a y LH $\beta$ de Pimelodus grosskopfii.

\begin{tabular}{|c|c|c|c|}
\hline Cebador & Secuencia & Accesión & Especie \\
\hline $1 \mathrm{GP}-\mathrm{a}$ & $\begin{array}{l}\text { 5'-CATTGGTGAAATTACTGTCCATTGC-3' } \\
\text { 5'-GAGATTTCTTGAACATACTAGTG-3' }\end{array}$ & KF934189 & Hemibagrus nemurus \\
\hline $2 \mathrm{GP}-\mathrm{a}$ & $\begin{array}{l}\text { 5'-GAAGAACAAAGACTGTCCATTGC-3' } \\
\text { 5'-GGGATTTCTTGAACATACAAGTC-3' }\end{array}$ & GQ889499 & Pelteobagrus fulvidraco \\
\hline $3 G P-a$ & $\begin{array}{l}\text { 5'-ATGATTCTTRTTCTCAAATACACTG-3' } \\
\text { 5'-TRAAAATTTATGYTAGTAGCAAGTG-3' }\end{array}$ & $\begin{array}{l}\text { KF934189 } \\
\text { GQ889499 }\end{array}$ & Hemibagrus nemurus \\
\hline $4 G P-a$ & $\begin{array}{l}\text { 5'-CACTGGAGCAACAATCCTTT-3' } \\
\text { 5'-TTCACTGATTGATACATAGTTGCAG-3' }\end{array}$ & NM_001200078 & Ictalurus punctatus \\
\hline $5 \mathrm{LH} \beta$ & $\begin{array}{l}\text { 5'-CCTGCTGAGCGATCACGGCAAAA-3' } \\
\text { 5'-GTGTGCAGCTCTTACAACAAGATC-3' }\end{array}$ & $\begin{array}{l}\text { KF573628 } \\
\text { KF934190 } \\
\end{array}$ & Heteropneustes fossilis \\
\hline $6 \mathrm{LH} \beta$ & \begin{tabular}{|l|} 
5'-ATGTCAGTGCCAGCTTTTTCTTTTC-3' \\
5'-TCAGTAGTCCAGGATAAACTCTTTC-3'
\end{tabular} & $\begin{array}{l}\text { NM_001200080 } \\
\text { KF934190 }\end{array}$ & Ictalurus punctatus \\
\hline 7LH $\beta$ & $\begin{array}{l}\text { 5'-ATGCCAGGTTCCTCTTTCCTTCT-3' } \\
\text { 5'-TCAGTAGTCCAGGATAAACTCTTTC-3' }\end{array}$ & $\begin{array}{l}\text { KF573628 } \\
\text { NM_001200080 }\end{array}$ & $\begin{array}{l}\text { Heteropneustes fossilis } \\
\text { Ictalurus punctatus }\end{array}$ \\
\hline $8 \mathrm{LH} \beta$ & \begin{tabular}{|l} 
5'-ATGCCTGCATCCTCTTACATACT-3' \\
5'-TCAGTAATCCAGGATGAACTCTTTC-3'
\end{tabular} & $\begin{array}{l}\text { KF934190 } \\
\text { KF573628 } \\
\end{array}$ & $\begin{array}{l}\text { Hemibagrus nemurus } \\
\text { Heteropneustes fossilis }\end{array}$ \\
\hline $9 L H \beta$ & $\begin{array}{l}\text { 5'-ATGCCWGCWTCCTCTWACAWACTTGA-3' } \\
\text { 5'-ATGCCTGSATCCTCTTAMATACTTGA-3' } \\
\text { 5'-TCAGTAGTCCAGYATAAACTCYTTC-3' }\end{array}$ & $\begin{array}{l}\text { AY973946 } \\
\text { KF573628 }\end{array}$ & $\begin{array}{l}\text { Silurus meridionalis } \\
\text { Heteropneustes fossilis }\end{array}$ \\
\hline $10 \mathrm{LH} \beta$ & $\begin{array}{l}\text { 5'-CCKGCTGAGCGAKCACTGCAAA-3' } \\
\text { 5'-GRYWGCAGRTCTTACAACAAGYTC-3' } \\
\text { 5'-GRGTGCAGRTCTTACAACAAGYTC-3' } \\
\text { 5'-GRGEGCAGRTCTTACAACAAGATC-3' }\end{array}$ & $\begin{array}{l}\text { KF934190 } \\
\text { KF573628 }\end{array}$ & $\begin{array}{l}\text { Hemibagrus nemurus } \\
\text { Heteropneustes fossilis }\end{array}$ \\
\hline $11 \mathrm{LH} \beta$ & $\begin{array}{l}\text { 5'-CCGGCTGAGCGATCACTGCAAAC-3' } \\
\text { 5'-GCAAGCAGTTCTTACAACAAGGTC-3' }\end{array}$ & $\begin{array}{l}\text { KF934190 } \\
\text { KF573628 }\end{array}$ & $\begin{array}{l}\text { Hemibagrus nemurus } \\
\text { Heteropneustes fossilis }\end{array}$ \\
\hline 12GP-a & $\begin{array}{l}\text { 5'-GCCACGTGATGATTCTTRTTCTCAAATACACTG-3' } \\
\text { 5'-GCGCGGCCGCTRAAATTTATGYTAGTAGCAAGTG-3' } \\
\text { 5'- GCGCGGCCGCAATTTATGYTAGTAGCAAGTG-3' }\end{array}$ & - & (2) \\
\hline $13 \mathrm{LH} \beta$ & $\begin{array}{l}\text { 5'-GCGAATTCATGCCAGGTTCCTCTTTCCTTCT-3' } \\
\text { 5'-GCTCTAGATCAGTAGTCCAGGATAAACTCTTTC-3' } \\
\text { 5'-GCTCTAGAGTAGTCCAGGATAAACTCTTTC-3' }\end{array}$ & - & - \\
\hline
\end{tabular}

\section{Análisis estructural y filogenético}

La predicción de las secuencias de aminoácidos para GP- $\alpha$ y LH $\beta$ fueron obtenidas con el programa SignalP versión 4.1. Para predecir la secuencia del péptido señal y los sitios putativos ligados para N-glicosilación se utilizó el programa NetNGlyc 1.0.

Las distancias genéticas, se calcularon mediante comparación por pares usando 1.000 repeticiones de bootstrap con el modelo de Kimura 2-parametros y se construyó un árbol a partir del método Neighbor-Joining usando el programa Mega versión 6.0.

\section{RESULTADOS}

El producto amplificado con los cebadores 3GP-a fue de 370 pb. Para estos cebadores las temperaturas óp- timas de alineamiento estuvieron entre 48 y $55,5^{\circ} \mathrm{C}$. Temperaturas superiores afectaron la eficiencia de la PCR. Por otro lado, con los cebadores 4GP-a se obtuvo un producto de $577 \mathrm{pb}$ con temperaturas óptimas de alineamiento entre 49,1 y $52,7^{\circ} \mathrm{C}$. El fragmento amplificado con los cebadores $7 \mathrm{LH} \beta$ fue de $417 \mathrm{pb}$.

La longitud del marco abierto de lectura para la secuencia de la subunidad GP-a fue de 351 pb, el cual incluyó un codón de inicio ATG y codón de parada TAA (accesión de GenBank KY860907). Por su lado, la longitud del marco abierto de lectura de la subunidad $\mathrm{LH} \beta$, fue de $423 \mathrm{pb}$, que incluyó un codón de inicio ATG y codón de parada TGA codificando para un péptido de 140 residuos de aminoácidos (aa). (cuadro 2). En GP-a, se identificó un péptido señal de 24 aa (MILVLKYTGATILLLSVLMDIGQL), dejando una proteína madura de 92 aa, 10 residuos de cisteína así como dos sitios de $\mathrm{N}$-glicosilación NIT y NHT (posiciones $\mathrm{Asn}^{53}$ y $\mathrm{Asn}^{78}$, 
figura 1). En LHß, se observó un péptido señal de 22 aa (MPGSSFLLVLCFLVNVFSPAQS), para una proteína madura de 118 aa, 12 residuos de cisteína y un sitio de $\mathrm{N}$-glicosilación NET (posición Asn ${ }^{10}$, figura 2)

El tamaño del péptido de GP-a de $P$. grosskopfii y sus características son similares a lo reportado para otras especies de Siluriformes como Clarias gariepinus, Silurus meridionalis y Hemibragus nemurus (ver la figura 1 y el cuadro 3). La presencia de dos sitios de N-glicosilación (posición $\mathrm{Asn}^{53}$ y $\mathrm{Asn}^{78}$ ) y 10 residuos de cisteína son altamente conservadas entre teleósteos y otros vertebrados [15] debido probablemente a que GP-a es compartido por todas las glicoproteínas pituitarias, incluyendo FSH, LH y Tirotropina (TSH). Se ha sugerido que estas regiones, tienen diferentes funciones, entre ellas, generar la formación de cinco enlaces disulfuro intra-moleculares relevantes para síntesis del péptido maduro y posiblemente relacionados con la dirección del dímero en la proteína [16]. Entre las GP- $a$ de diferentes especies de peces, se identifica una región altamente conservada entre los residuos de aminoácidos 33-66, ubicada entre dos pares de cisteínas adyacentes y el primer sitio de $\mathrm{N}$-glicosilación, que puede estar involucrada en el ensamble de la subunidad y su afinidad para unirse al receptor [17].

La longitud de la proteína madura de LH $\beta$ de $P$. grosskopfii de 118 aa está estrictamente conservada entre las especies del orden siluriformes incluidas en el presente análisis (figura 2). En otros grupos de teleósteos en los cuales LH $\beta$ ha sido clonada, se ha determinado que las longitudes de la proteína madura y el péptido señal varían entre 115-119 y 21-27 aa respectivamente $[18,19]$. El análisis de la topología de la estructura del péptido maduro de LH $\beta$ en $P$. grosskopfii, indicó la presencia de un sitio de $\mathrm{N}$-glicosilación (posición $\mathrm{Asn}^{10}$ ) y 12 residuos de cisteína. Esta estructura es conservada en las diferentes secuencias de LH $\beta$ previamente reportadas en teleósteos (figura 2). Adicionalmente, se identificó una región ampliamente conservada (HPGVDPHVTYPVALS) que concentra residuos de prolinas, que son esenciales para la estructura proteica de la subunidad $\mathrm{LH} \beta$ debido a su importancia en el plegamiento de la proteína y direccionamiento del péptido [20].

Figura 1. Alineamiento de la estructura primaria del péptido de GP-a de P. grosskopfii con otros teleósteos

\begin{tabular}{|c|c|}
\hline Oreochromis_niloticus & -MGSLKSPGLSLLLLSFLLYIADSYPNIDLSNMGCEECTLRKNNLFSR-DRPVYOCMGCC \\
\hline Oncorhynchus_mykiss & -MCLLKSTGL-FLILSALLVIADSYPNSDKTNMGCEECFLKPNTIFPN----IMdCFdC \\
\hline Arapaima gigās & MSYTGKLTIASVLALLAILHIVDSNFN-----VGCEE|KLKENKYFSRLG \\
\hline Danio_rerio & -MFWTRYAEASIFLLLMILHVGQLYSRNDVSNYGCEECKLKMNERFSKP \\
\hline Carass̄ius_auratus & -MEWTRYAGASILLFLMLIHLGQLYPRNYMNNFGCEECELKENNI \\
\hline Astyanax_zaltiparanae & MILIIKYTGAAVLLLSVLIQIGQLYPHNDMY-FGCEDCKLRENSI \\
\hline ilurus_meridionalis & MTLIPKYTGATIVLLSVLIEVGQPYPNND---FGCEECKLKENSI \\
\hline Colossoma_macropomum & MILIIKYAGATILLLSVLIQTGQLYLNNDITNFGCEECKLKENNIE \\
\hline Pimelodus grosskopfii & MILVLKYTGATILLLSVLMDIGQLYPNND---FGCEECRLRENNIFSKPG \\
\hline Clarias gariepinus & MTLIPKYTGATILLLCVLIEIGQLYPNND---FGCEECKLKENNIESKPG \\
\hline Hepteroñeutes_fossilis & MTLILKYTGATVLLLCGLIEIGQLYE \\
\hline Ictalurus_punc̄tatus & MILILKYTGATI ILLSVLIEIGQLFPNND---FGCEECKLKENN I \\
\hline Hemibagrus_nemurus & MILILKYTGATIELLSVLIEIGQLYPNND---EGCEECKLKENNI \\
\hline Pelteobagrüs_fulvidraco & MILVLKYTGATIFLLSVLIEIGQLYPNND---FGCEECKLKENN I \\
\hline & $\cdot \quad \cdot \quad \cdots \quad: \quad::$ \\
\hline Oreochromis_niloticus & FSRAYPTPLRATKTMNIPKNITSEATCFNARHSYEIE-IAGIKVRMHTDC \\
\hline Oncorhynchus_mykiss & FSRAYPTPLRSKQTMLVPKNITSEATCCVAKEGERVTTKDGFPVT \\
\hline Arapaima_gigās & FSRAYPTPLRSKKTMLVPKNITSEATCENAKEVKRLITLNNVRLE \\
\hline Danio_rerio & FSRAYPTPLRSKKTMLVPKNITSEATCEVAKE-SKMV-ATNIPLY \\
\hline Carassius_auratus & FSRAYPTPLRSKKTMLVPKNITSEATCENAKEVKRVL-VNDVRLV \\
\hline Astyanax āltiparanae & FSRAYPTPLRSKKTMLVPKNITSEATCCVAKEIQRVF-VNNVKLolHTDC \\
\hline Silurus_meridionalis & FSRAYPTPLRSKETMLVPKNITSEATCCVAKEFKRVI-VNDVKLI \\
\hline Colossoma_macropomum & FSRAYPTPLRSKKTMLVPKNITSEAT|CCVAKEFKRVI-VNDVKLV- \\
\hline Pimelodus grosskopfii & FSRAYPTPLRSKKTMLVPK ITSEATCCNAKEVKRVI-VNDVKLV IHTDCHCISTCYYHKS \\
\hline Clarias_gariepinus & FSRAYPTPLRSKKTMLVPKNITSEATCEVAKEVKRVI-VNDVKLVAHTDCF \\
\hline Hepteroñeutes_fossilis & FSRAYPTPLRSKKTMLVPKNITSEATCEVAKEVKRVI-VNDVKLV- \\
\hline Ictalurus_punc̄tatus & FSRAYPTPLRSEKTMLVPKNITSEATCEVAKEVKRVI-VNDVKLM \\
\hline Hemibagruśnemurus & FSRAYPTPLRSKKTMLVPKNITSEATCEVAKEVKRVI-VNDVKLVAHTDCHCSTCYYHKF \\
\hline Pelteobagrūs_fulvidraco & FSRAYPTPLRSKKTMLVPKNITSEATCCVAKEVKRVI-VNDVKLVUHTDCHCSTCYYHKF \\
\hline
\end{tabular}

Los residuos de cisteína son identificados con cuadros. Los sitios de N-glicosilación son identificados con cuadros en sombra. Para el análisis se incorporaron los espacios (gaps) marcados con (-). 
Cuadro 2. Secuencia nucleotídica y predicción de aminoácidos de GP-a y LH $\beta$ de Pimelodus grosskopfii.

\begin{tabular}{|c|c|}
\hline Secuencia Nucleótidos GP- $a$ & $\begin{array}{l}\text { ATGATTCTTGTTCTCAAATACACTGGAGCAACAATTCTTTTACTGTCTGTTTTAATGGATA- } \\
\text { TTGGACAACTTTACCCAAATAATGACTTTTGGCTGTGAAGAGTGCAAACTCAGGGAGAA- } \\
\text { CAATATTTTCTCAAAACCTGGCGCTCCTGTGTATCAGTGCATGGGATGCTGCTTTTCCA- } \\
\text { GAGCTTACCCGACTCCACTGAGATCCAAGAAGACTATGCTGGTTCCGAAGAATATTA- } \\
\text { CATCTGAAGCCACATGTTGCGTTGCCAAAGAGGTTAAACGGGTAATCGTTAATGATG- } \\
\text { TAAAGCTAGTGAATCACACAGACTGCCACTGCAGCACTTGCTACTATCATAAATCTTAA }\end{array}$ \\
\hline $\begin{array}{l}\text { Predicción secuencia aminoácidos } \\
\text { GP-a }\end{array}$ & $\begin{array}{l}\text { MILVLKYTGATILLLSVLMDIGQLYPNNDFGCEECKLRENNIFSKPGAPVYQCMGCCFS- } \\
\text { RAYPTPLRSKKTMLVPKNITSEATCCVAKEVKRVIVNDVKLVNHTDCHCSTCYYHKS- }\end{array}$ \\
\hline Secuencia Nucleótidos LH $\beta$ & $\begin{array}{l}\text { ATGCCAGGTTCCTCTTTCCTTCTTGTCCTATGTTTTTTGGTCAACGTCTTTCTCCTGCT- } \\
\text { CAAAGCTTCCTTCTGACCCACTGTGAACCTGTTAATGAGACTGTCTCTGTGGAGAAAGAT- } \\
\text { GGCTGCCCAAGATGCCTTGTGTTTCAAACCACCATCTGCAGTGGACACTGCTTCACCAAG- } \\
\text { GAACCTGTGTACAAGAGCGCGTTCTCTTCCATCTATCAGCATGTGTGCACCTACAGAGAC- } \\
\text { GTTCGCTACGAAACGGTTCGCCTGCCAGACTGTCACCCAGGGGTGGATCCCCATGTCACC- } \\
\text { TATCCTGTCGCTCTAAGTTGCGAGTGCAGCTTGTGCACCATGGACACCTCGGACTGTAC- } \\
\text { CATCGAGAGCCTGAATCCGGATTTCTGTATGACACAGAAAGAGTTATCCTGGACTACTGA }\end{array}$ \\
\hline $\begin{array}{l}\text { Predicción secuencia aminoácidos } \\
\text { LH } \beta\end{array}$ & $\begin{array}{l}\text { MPGSSFLLVLCFLVNVFSPAQSFLLTHCEPVNETVSVEKDGCPRCLVFQTTICSGHCF- } \\
\text { TKEPVYKSAFSSIYQHVCTYRDVRYETVRLPDCHPGVDPHVTYPVALSCECSLCTMDTS- } \\
\text { DCTIESLNPDFCMTQKEFILDY- }\end{array}$ \\
\hline $\begin{array}{l}\text { Se señalan el Codón de inicio y parada, } \\
\text { Señal del péptido, (-) aminoácido de } \\
\text { terminación, Sitio de } N \text { - glicosilación }\end{array}$ & \\
\hline
\end{tabular}

Cuadro 3. Homología de nucleótidos y predicción de aminoácidos para GP-a y LHß de Pimelodus grosskopfii.

\begin{tabular}{|c|c|c|c|c|c|c|}
\hline \multirow{2}{*}{ Orden } & \multirow{2}{*}{ Accesión GenBank } & \multirow{2}{*}{ Especie } & \multicolumn{2}{|c|}{$\%$ identidad nucleótidos } & \multicolumn{2}{|c|}{ \% identidad aminoácidos } \\
\hline & & & GP- $a$ & LH $\beta$ & GP- $a$ & LH $\beta$ \\
\hline \multirow[t]{2}{*}{ Siluriformes } & $\begin{array}{l}\text { KF934189 } \\
\text { KF934190 }\end{array}$ & Hemibagrus nemurus & 92 & 91 & 94 & 91 \\
\hline & $\begin{array}{l}\text { X97760.1 } \\
\text { X97761.1 }\end{array}$ & Clarias gariepinus & 91 & 88 & 93 & 89 \\
\hline \multicolumn{2}{|c|}{ MF373412.1 } & Clarias major & 91 & - & 92 & - \\
\hline & KM258876.1 & Clarias batrachus & - & 89 & - & 89 \\
\hline & $\begin{array}{l}\text { GQ889499.1 } \\
\text { KP036409.1 }\end{array}$ & Tachisurus fulvidraco & 91 & 89 & 95 & 88 \\
\hline & $\begin{array}{c}\text { NM_001200078 } \\
\text { AF112192 }\end{array}$ & Ictalurus punctatus & 90 & 88 & 92 & 89 \\
\hline & $\begin{array}{l}\text { AY973945.1 } \\
\text { AY973946.2 }\end{array}$ & Silurus meridionalis & 86 & 85 & 86 & 92 \\
\hline & $\begin{array}{c}\text { KF573626 } \\
\text { KF573626.1 }\end{array}$ & Hepteroneutes fossilis & 91 & 89 & 92 & 91 \\
\hline \multirow{3}{*}{ Cypriniformes } & XM_019096146.1 & Cyprinus carpio & 80 & 73 & 86 & 73 \\
\hline & $\begin{array}{c}\text { AY800266 } \\
\text { D88024 }\end{array}$ & Carassius auratus & 80 & 74 & 86 & 73 \\
\hline & $\begin{array}{c}\text { AY424306 } \\
\text { AY424304.1 }\end{array}$ & Danio rerio & 73 & 70 & 71 & 65 \\
\hline Salmoniformes & $\begin{array}{c}\text { AB050836 } \\
\text { NM001124205.1 }\end{array}$ & Oncorhynchus mykkis & 72 & 68 & 75 & 65 \\
\hline Perciformes & $\begin{array}{l}\text { NP_001266680.1 } \\
\text { XP_003438397.1 }\end{array}$ & Oreochromis niloticus & 65 & 65 & 64 & 59 \\
\hline \multirow{2}{*}{ Characiformes } & $\begin{array}{c}\text { KU312261 } \\
\text { KU312262.1 }\end{array}$ & Colossoma macropomum & 85 & 80 & 92 & 84 \\
\hline & $\begin{array}{l}\text { KJ544555.1 } \\
\text { KJ544556 }\end{array}$ & Astyanax altiparanae & 80 & 79 & 85 & 77 \\
\hline Osteoglossiformes & $\begin{array}{l}\text { KJ755358 } \\
\text { KJ741848 }\end{array}$ & Arapaima gigas & 68 & 68 & 71 & 66 \\
\hline
\end{tabular}


Figura 2. Alineamiento de la estructura primaria del péptido de LH $\beta$ de P. grosskopfii, con otros teleósteos.

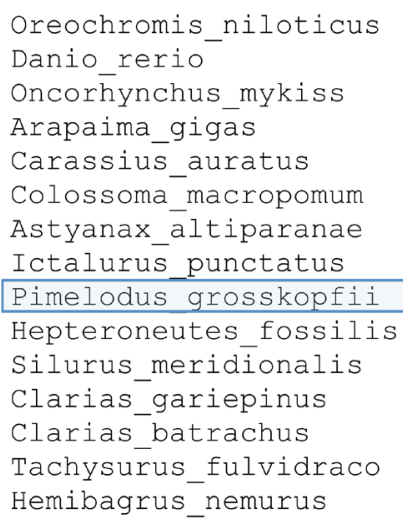

Los residuos de cisteína son identificados con cuadros. Los sitios de N-glicosilación son identificados con cuadros en sombra. Para el análisis se incorporaron los espacios (gaps) marcados con (-).

Se encontró una alta homología de las secuencias de nucleótidos (86-92\%) y aminoácidos (86-95\%) de la subunidad GP-a de P. grosskopfii con otros géneros de Siluriformes como: Hemibragrus, Clarias, Pelteobragus, Ictalurus, Tachisurus y Silurus. En contraste, se evidenció menor homología de la subunidad GP-a de P. grosskopfii con peces de otros ordenes como Characiformes, Cypriniformes, Salmoniformes, Osteoglossiformes y Perciformes a nivel de nucleótidos (65-85\%) y para la predicción de aminoácidos (64-92\%) (cuadro 3). Igual- 
mente, para LH $\beta$ de $P$. grosskopfii se registró una alta homología de la secuencia de nucleótidos (8591\%) y aminoácidos (88-92\%) con otros Siluriformes así como menor homología con peces de los órdenes Characiformes, Cypriniformes, Salmoniformes, Osteoglossiformes y Perciformes a nivel de nucleótidos (65-80\%) y para la predicción de aminoácidos (59-84\%) (cuadro 3).

La filogenia basada en las secuencias de LH $\beta$ separó claramente los diferentes órdenes incluidos en el análisis, comparado con el árbol basado en GP-a (figuras 3 y 4) ya esta última filogenia no agrupó en un solo clado a los miembros del órden Characiformes. Sin embargo, en ambas filogenias los miembros del orden Siluriformes presentaron menor distancia entre sí, comparados con representantes de Osteoglossiformes, Salmoniformes y Perciformes. En ambos árboles, los bagres del viejo mundo (Silurus, Heteropneustes, Clarias, Hemibragrus y Tachisurus) fueron más cercanos entre sí, comparados con uno de los dos bagres americanos (Ictalurus o Pimelodus) según la reconstrucción filogenética analizada.

En general las relaciones presentadas en la filogenia basada en las secuencias de LH $\beta$ fueron consistentes con los resultados obtenidos por [21,22] para la clase Actinopterygii ya que se identificó a: 1. Osteoglossomorpha (Arapaima gigas) como el linaje hermano de los demás teleósteos analizados y 2. el clado Otophysi (compuesto por Characiformes y Siluriformes como grupos hermanos junto a Cypriniformes). La principal diferencia entre la filogenia basada en $\mathrm{LH} \beta$ y las propuestas por [21,22] fue la relación de Salmoniformes
Figura 3. Relaciones filogenéticas de la subunidad GP- $a$ de P. grosskopfii con otras de especies teleósteos.

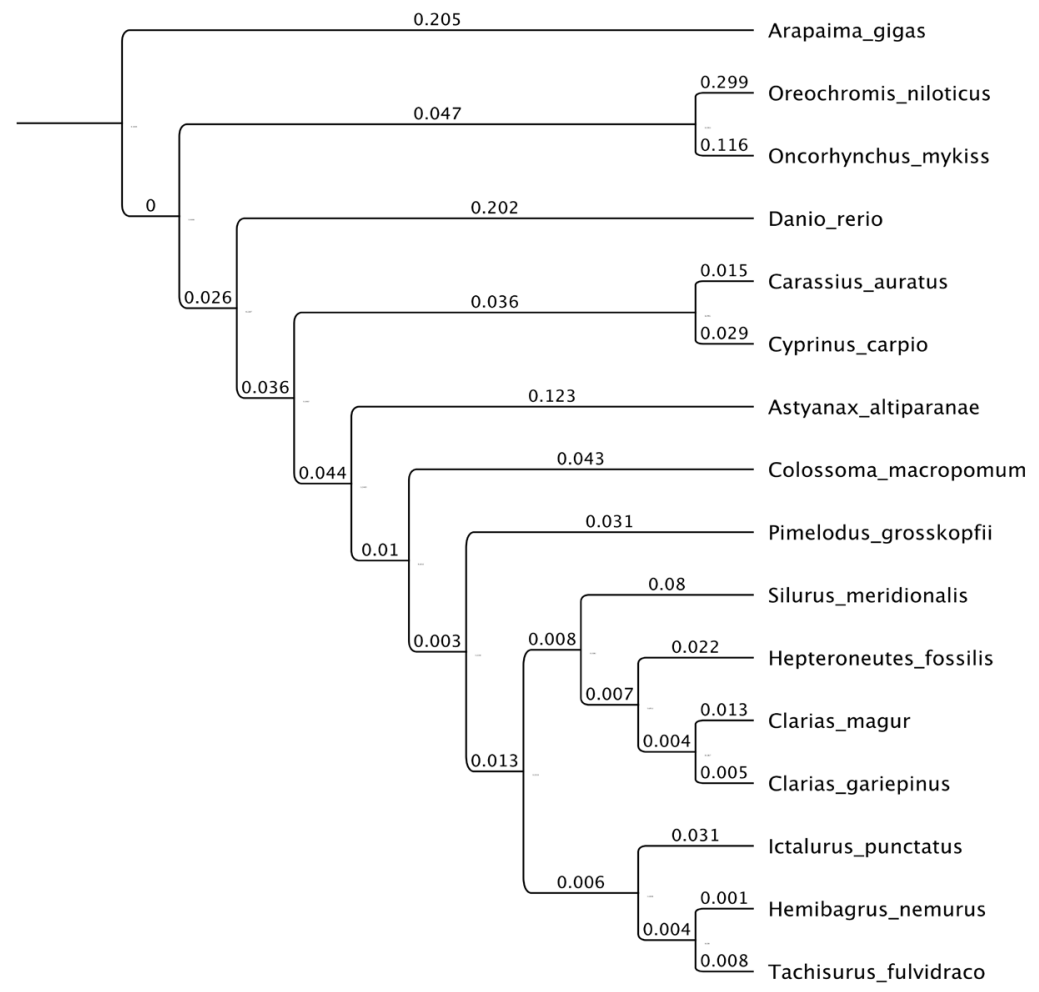

Figura 4. Relaciones filogenéticas de la subunidad LH $\beta$ de P. grosskopfii con otras de especies teleósteos.

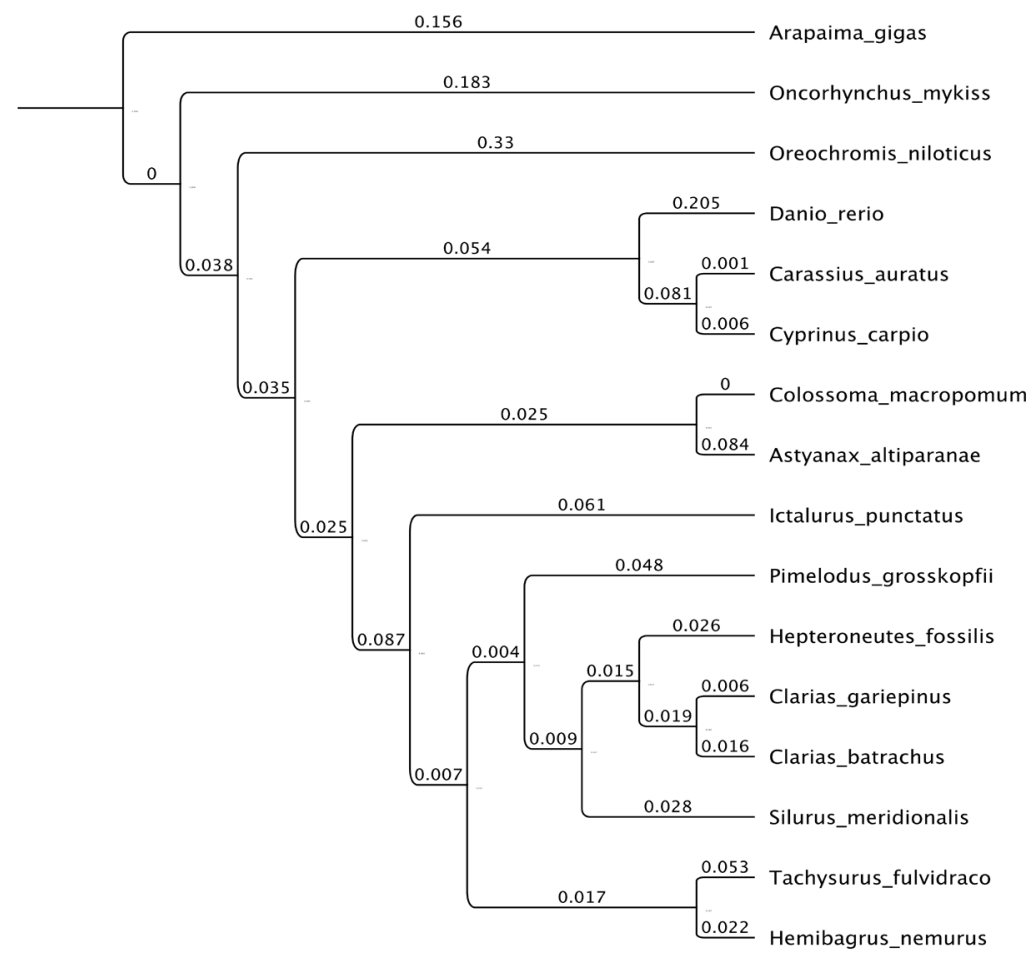


y Perciformes sin la formación del clado Euteleostei que si se presentó en la filogenia basada en GP-a. Las relaciones entre las familias del orden Siluriformes encontradas en las filogenias basadas en GP-a o LH $\beta$ conservaron la relación entre Hepteroneustes con Clarias (Claroidea), Tachysurus con Hemibagrus (Bagridae) y Silurus (Siluridae) como linaje independiente que se presentan en [23]. La relación de Ictalurus con Bagridae observada en la filogenia basada en GP-a fue consistente con la filogenia presentada por [22] pero fue diferente a la obtenida para LH $\beta$. La posición de Pimelodidae en las filogenias basadas en GP- $\alpha$ o LH $\beta$ es diferente a lo descrito por [22] debido a que estos autores encontraron a Pimelodidae como parte del grupo hermano del clado del que hacen parte Ictaluridae más Bagridae

\section{CONCLUSIONES}

El presente estudio, es el primer reporte de las secuencias de las subunidades de una gonadotropina en una especie de la familia Pimelodidae. El marco abierto de lectura para las secuencias de las subunidades GP- $\alpha$ y LH $\beta$ de $P$. grosskopfii fue de 351 y 423 pb respectivamente. En GP-a, se identificó un péptido señal de 24 aa dejando una proteína madura de 92 aa. Por otra parte, en LH $\beta$, se observó un péptido señal de 22 aa para una proteína madura de 118 aa. La homología de las secuencias de nucleótidos y aminoácidos de GP- $\alpha$ y LH $\beta$ de $P$. grosskopfii fue mayor con miembros del orden Siluriformes comparados con otros ordenes de peces. Las filogenias construidas a partir de GP-a o $\mathrm{LH} \beta$ conservan muchas de las relaciones previamente reportadas entre los grandes grupos de peces con aletas radiadas, pero presentan particularidades, entre ellas la posición de Pimelodus al interior de Siluriformes. Estos resultados son un punto de partida para el desarrollo de anticuerpos para cuantificación de hormonas gonadotrópicas y la producción de hormona luteinizante recombinante en $P$. grosskopfii.

\section{AGRADECIMIENTOS}

Esta investigación fue financiada por la Secretaria de Agricultura y Desarrollo Rural de la Gobernación de Antioquia en el marco del convenio 4600000970 SADRA-ASOACUICOLA del fondo de Ciencia Tecnología e innovación del Sistema General de Regalías.

\section{REFERENCIAS}

[1] YOM-DIN, S. et al. Gonadotropins in the Russian sturgeon: Their role in steroid secretion and the effect of hormonal treatment on their secretion. PLoS ONE, 11, 2016, p. e0162344. doi:https:// doi.org/10.1371/journal.pone.0162344

[2] BUROW, S. et al. Medaka follicle-stimulating hormone (Fsh) and luteinizing hormone (Lh): Developmental profiles of pituitary protein and gene expression levels. General and Comparative Endocrinology, 272, 2019, p. 93-108. doi:https://doi.org/10.1016/j.ygcen.2018.12.006

[3] KANDA, S. Evolution of the regulatory mechanisms for the hypothalamic-pituitary-gonadal axis in vertebrates-hypothesis from a comparative view. General and Comparative Endocrinology, 27, 2018, p. S0016-6480(18)30494-5.doi: https://doi.org/10.1016/j.ygcen.2018.11.014

[4] LI, J. and CHENG, C. Evolution of gonadotropin signaling on gonad development: insights from gene knockout studies in zebrafish. Biology of Reproduction, 99, 2018, p. 686-94. doi:https:// doi.org/10.1093/biolre/ioy101/4987199

[5] SHANG, G. et al. Steroidogenic acute regulatory protein and luteinizing hormone are required for normal ovarian steroidogenesis and oocyte maturation in zebrafish. Biology of Reproduction, ioz132, 2019. doi: https://doi.org/10.1093/biolre/ioz132

[6] LUNDBERG, J., SULLIVAN, J.P. and HARDMAN, M. Phylogenetics of the South American catfish family Pimelodidae (Teleostei: Siluriformes) using nuclear and mitochondrial gene sequences. Proceedings of the Academy of Natural Sciences of Philadelphia, 161, 2011, p. 153-89. doi: https://doi.org/10.2307/41446000

[7] VILLA-NAVARRO, F.A., ACERO, A. and CALA, P.C. Taxonomic review of Trans-Andean species of Pimelodus (Siluriformes: Pimelodidae), with the descriptions of two new species. Zootaxa, 4299, 2017, p. 337-60. doi: https://doi. org/10.11646/zootaxa.4299.3.2

[8] GARCÍA-DÁVILA, C. et al. Using barcoding of larvae for investigating the breeding seasons of pimelodid catfishes from the Marañon, Napo and Ucayali rivers in the Peruvian Amazon. Journal of Applied Ichthyology, 31, 2015, p. 40-51. doi. https://doi.org/10.1111/jai.12987 
[9] FREITAS, T.M. and MONTAG, L.F. Population and reproductive parameters of the red-tailed catfish, Phractocephalus hemioliopterus (Pimelodidae: Siluriformes), from the Xingu River, Brazil. Neotropical Ichthyology, 17, 2019, p. 1-8. doi: https:// doi.org/10.1590/1982-0224-20190015

[10] DOS SANTOS, J.E. et al. Reproductive biology of the Neotropical catfish Iheringichthys labrosus (Siluriformes: Pimelodidae), with anatomical and morphometric analysis of gonadal tissues. Animal Reproduction Science, 209, 2019, p. 106173. doi: https://doi.org/10.1016/j.anireprosci.2019.106173

[11] DAMASCENO, D.Z. et al. Induced spermiation of Pimelodus britskii (Teleostei : Pimelodidae) during the reproductive period. Aquaculture Research, 2015, p. 1-13. doi: https://doi.org/10.1111/are.12930

[12] HONJI, R.M., CANEPPELE, D. and PANDOLFI, $M$. Gonadotropins and growth hormone family characterization in an endangered siluriform species, Steindachneridion parahybae (Pimelodidae): relationship with annual reproductive cycle and induced spawning in captivity. Anatomical Record, 1658, 2015, p. 1644-58. doi: https://doi. org/10.1002/ar.23174

[13] FRICKE, R., ESCHMEYER, W. and VAN DER LAAN, R. Eschmeyer's catalog of fishes: genera, species [online]. 2019. Disponible: http://researcharchive. calacademy.org/research/ichthyology/catalog/fishcatmain.asp [citado 14 de octubre de 2019]

[14] VILLA-NAVARRO, F. et al. Pimelodus grosskopfii The IUCN Red List of Threatened Species [online]. 2016. Disponible: https://www.iucnredlist. org/species/49829828/61473588 [citado 14 de octubre de 2019]

[15] PRADHAN,A, RATH S.C and SAHA, A. Molecular characterization and expression analysis of gonadotrophic a-subunit hormone in rohu (Labeo rohita). International Journal of Fisheries and Aquatic Studies, 5, 2017, p. 23-28.

[16] LARSON, S.B. and MCPHERSON, A. The crystal structure of the $\beta$ subunit of luteinizing hormone and a model for the intact hormone. Current Research in Structural Biology, 1, 2019, p. 1-5. doi: https://doi.org/10.1016/j.crstbi.2019.07.001

[17] DE JESUS, L.W. et al. Gonadotropin subunits of the characiform Astyanax altiparanae: molecular characterization, spatio temporal expression and their possible role on female reproductive dysfunction in captivity. General and Comparative Endocrinology, 246, 2017, p. 150-63. doi. https:// doi.org/10.1016/j.ygcen.2016.12.004
[18] RATHER, M.A., BHAT, I.A. and SHARMA, R. Identification, cDNA cloning, and characterization of luteinizing hormone beta subunit (Lhb) gene in Catla catla. Animal Biotechnology, 27, 2016, p. 148-56. doi: https://doi.org/10.1080/1049539 8.2016.1140055

[19] GAO, Y. et al. Molecular cloning, characterization, and mRNA expression of gonadotropins during larval development in turbot (Scophthalmus maximus). Fish Physiology and Biochemistry, 45, 2019, p. 1697-1707. doi: https://doi.org/10.1007/s10695019-00656-Z

[20] SEVILHANO, T. et al. Molecular cloning and characterization of pirarucu (Arapaima gigas) follicle-stimulating hormone and luteinizing hormone $\beta$-subunit cDNAs. PLoS ONE, 12, 2017, p. e0183545. doi: https://doi.org/10.1371/journal.pone.018354

[21] NEAR, T.J. et al. Resolution of ray-finned fish phylogeny and timing of diversification. Proceedings of the National Academy of Sciences of the United States of America, 109, 2012, p. 13698-13703. doi: https://doi.org/10.1073/pnas.1206625109

[22] BETANCUR, R. et al. Phylogenetic classification of bony fishes. BMC Evolutionary Biology, 17, 2017, p. 162. doi: https://doi.org/10.1186/ s12862-017-0958-3

[23] SULLIVAN, J.P., LUNDBERG, J.G. and HARDMAN, $M$. A phylogenetic analysis of the major groups of catfishes (Teleostei: Siluriformes) using rag1 and rag2 nuclear gene sequences. Molecular Phylogenetics and Evolution, 41, 2006, p. 636-662. doi. https://doi.org/10.1016/j.ympev.2006.05.044 